\title{
Flax Inorganic Phosphate Deficiency Responsive miRNAs
}

\author{
Nataliya V. Melnikova ${ }^{1}$, Maxim S. Belenikin ${ }^{1,2}$, Nadezhda L. Bolsheva ${ }^{3}$ Alexey A. Dmitriev ${ }^{4}$, Anna S. \\ Speranskaya $^{1,5}$, Anastasia A. Krinitsina ${ }^{5}$, Nadezda V. Koroban ${ }^{1}$, Tatiana E. Samatadze ${ }^{3}$, Alexandra V. \\ Amosova $^{3}$,Olga V. Muravenko ${ }^{3}$, Alexander V. Zelenin ${ }^{3} \&$ Anna V. Kudryavtseva ${ }^{1}$ \\ ${ }^{1}$ Group of Post-Genomic Research, Engelhardt Institute of Molecular Biology, Russian Academy of Sciences, \\ Moscow, Russia \\ ${ }^{2}$ Laboratory of Bioinformatics, Research Institute of Physicochemical Medicine, 119435 Moscow, Russia \\ ${ }^{3}$ Laboratory of Molecular Karyology and Basics of Cell Therapy, Engelhardt Institute of Molecular Biology, \\ Russian Academy of Sciences, Moscow, Russia \\ ${ }^{4}$ Laboratory of Structural and Functional Genomics, Engelhardt Institute of Molecular Biology, Russian Academy \\ of Sciences, Moscow, Russia \\ ${ }^{5}$ Department of Higher Plants, Lomonosov Moscow State University, Moscow, Russia \\ Correspondence: Nataliya V. Melnikova, Group of Post-Genomic Research, Engelhardt Institute of Molecular \\ Biology, Moscow, Russia. Tel: 7-499-135-2391. E-mail: mnv-4529264@yandex.ru
}

Received: October 16, 2013 Accepted: November 7, 2013 Online Published: December 15, 2013

doi:10.5539/jas.v6n1p156 URL: http://dx.doi.org/10.5539/jas.v6n1p156

\begin{abstract}
MicroRNAs (miRNAs) control a variety of biological processes and are involved in plant response to abiotic stress including phosphate starvation. Phosphate deficiency is one of the major limiting factors for crop yield. In the present work inorganic phosphate deficiency responsive miRNAs were identified in flax for the first time by high-throughput sequencing. Three conserved miRNA families (miR398, miR399 and miR408) were differentially expressed under inorganic phosphate starvation. In other plant species it was demonstrated that these miRNAs are involved in response to a combination of different stresses. In phosphate starvation conditions miR399 and miR408 are up-regulated but miR398 is differentially expressed. We found that in flax under inorganic phosphate starvation stress conditions expression level alterations for conserved miRNAs miR399 and miR408 were close to those in other plant species and for miR398 - to those in tomato but not in Arabidopsis. A better understanding of miRNAs stress regulatory functions would provide new tools for genetic improvement of stress tolerance in flax and other plants.
\end{abstract}

Keywords: flax (Linum usitatissimum), microRNAs, inorganic phosphate, abiotic stress

\section{Introduction}

Flax is an important agricultural crop and is used as a source of stem fiber and seed oil. Flax fiber is used in textiles, paper, isolation material, composite materials. Flax oil is used in linoleum, varnishes, paints and plastics (Cullis, 2007). Some of flax lines are undergone heritable phenotype and genotype changes in response to defined environments including phosphate starvation. Changes in total DNA content, copy number variation of ribosomal RNA genes and satellite regions, the appearance of Linum Insertion Sequence 1, variations in plant height, weight, and branching, isozyme mobility, and capsule septa hair number were identified in flax line 'Stormont Cirrus' under stress conditions (Durrant, 1958; Durrant, 1962; Evans, Durrant, \& Rees, 1966; Cullis \& Charlton, 1981; Tyson \& Fieldes, 1982; Chen, Schneeberger, \& Cullis, 2005; Chen, Lowenfeld, \& Cullis, 2009; Bickel, Lukacs, \& Cullis, 2012). Very little is known about mechanisms of genetic and phenotypic changes regulation during flax development under stress conditions.

MicroRNAs (miRNAs) are a class of small non-coding RNAs that have been found in diverse organisms. In plants, the mature miRNAs are usually approximately 21-24 nucleotides in length. MiRNAs negatively regulate gene expression by specific binding and cleavage of mRNAs, or by mRNA translation inhibition (Jones-Rhoades, D. P. Bartel \& B. Bartel, 2006). In recent years, it has been found that miRNAs control a variety of biological processes, are essential for normal plant growth and development, and are involved in plant response to abiotic stress 
including phosphate starvation (Sunkar, Chinnusamy, J. Zhu, \& J. K. Zhu, 2007; Guleria, Mahajan, Bhardwaj, \& Yadav, 2011).

Phosphorus is one of the essential elements for plants. It is required for the synthesis of nucleic acids and membrane lipids, but it is often a limiting element for plant growth. Phosphate deficiency is one of the major limiting factors for crop yield. In this study, we sequenced flax (Linum usitatissimum L.) miRNAs with Illumina GAIIx and identified flax inorganic phosphate (Pi) deficiency responsive miRNAs for the first time. Our study of flax Pi deficiency responsive miRNAs may give us a better understanding of gene regulation in flax under stress conditions and the role of miRNA in this process.

\section{Material and Methods}

\subsection{Plant Materials}

Flax (L. usitatissimum L.) cultivar 'Stormont Cirrus' was planted in a climate chamber under a 16-hour light/8-hour dark cycle at $22^{\circ} \mathrm{C}$ in 5" pots with pearlite for six weeks. Plants were grown under control (C) nutrition (100 ml of $0.5 x$ Hoagland's Solution (Hoagland \& Arnon, 1950), pH5.5, was applied weekly) and the lack of Pi (P): $\left(100 \mathrm{ml}\right.$ of $0.5 \mathrm{x}$ Hoagland's Solution without $\mathrm{KH}_{2} \mathrm{PO}_{4}, \mathrm{pH} 5.5$, was applied every seven days; potassium concentration was compensated by potassium acetate buffer). The lack of Pi nutrition conditions were similar to those previously shown to result in heritable changes in Stormont Cirrus (Durrant, 1958, 1962; Cullis \& Charlton 1981).

\subsection{RNA Extraction}

Leaf samples were collected from individual plants after 6 weeks of growth and were ground in liquid nitrogen and either used immediately for RNA isolation or stored at $80^{\circ} \mathrm{C}$. Total RNA was extracted using ZR RNA MicroPrep reagents. RNA quantity was evaluated by Qubit 2.0, and RNA quality was evaluated by Agilent 2100 Bioanalyzer.

\subsection{Small RNA Sequencing}

High quality RNA samples were used for small RNA library preparation. L. usitatissimum small RNA cDNA library was constructed using the Illumina TruSeq small RNA sample preparation kit following the protocol described in the TruSeq Small RNA Sample Preparation Guide (www.illumina.com). Briefly, $1 \mu \mathrm{g}$ of total RNA was first ligated with specific 3' and 5 ' adaptors. After reverse transcription (SuperScript II Reverse Transcriptase, Invitrogen) and PCR amplification, cDNAs were purified using the 6\% PAGE Gel, then libraries were validated on an Agilent Technologies 2100 Bioanalyzer using a High Sensitivity DNA chip and sequenced on the Illumina GAIIx platform.

\subsection{Small RNA Bioinformatics Analysis}

Raw sequence reads were generated from the Illumina GAIIx and were converted into full-length reads by removing low-quality reads and adapter reads. For the next processing we used cleaned reads with abundance six or more times. To identify conserved known miRNAs in flax, sRNAs were aligned with known matured miRNAs sequences found in miRBase (Kozomara \& Griffiths-Jones, 2011).

\subsection{Expression Analysis of Flax miRNAs in Pi Deficiency}

Expression analyses of miRNAs were performed for sequence reads generated from the $\mathrm{P}$ and $\mathrm{C}$ nutrition libraries. Expression levels of miRNAs in the flax libraries were normalized to obtain the number of miRNAs per million reads.

If normalized expression level of a given miRNA was equal to 0 , then its expression value was modified to 0.01 . Normalized reads were used to calculate the changes in miRNAs expression. If the normalized expression level of a given miRNA was less than 1 in $\mathrm{C}$ and $\mathrm{P}$ libraries, then this miRNA was removed from further analysis.

Fold changes were calculated using the following formula:

$$
\text { fold change }=\log _{2} \text { (expression level in Pi deficiency/expression level in control nutrition) }
$$

$P$-values were obtained according to the calculations of (Audic \& Claverie, 1997).

\section{Results and Discussion}

\subsection{Identification of Phosphate Deficiency Responsive Flax miRNA}

Small RNAs of flax grown in Pi deficiency were sequenced for the first time. A total over 7 million (control nutritious) and over 11 million (Pi deficiency) raw reads were obtained from deep sequencing of flax sRNA with genome analyzer Illumina GAIIx. 
We compared flax miRNA candidates with miRNAs identified in other plant species using miRNA database miRBase (www.mirbase.org). As a result, we identified conserved miRNA families in flax and among them we found three that were differentially expressed (Table 1). Expression levels of the miRNAs were analyzed by normalized reads (reads number per million genome-matched reads - RPM). Some of the miRNA families showed differential expression among $\mathrm{C}$ and $\mathrm{P}$ flax libraries: miR398 (2.0-fold change; $p$-value $\left.1.5^{*} 10^{-11}\right)$, miR399 (2.2-fold change; $p$-value 0.02 ) and miR408 (2.3-fold change; $p$-value $\left.6.6^{*} 10^{-7}\right)$.

Table 1. Expression levels of differentially expressed miRNAs in flax under control nutrition and Pi deficiency

\begin{tabular}{cccc}
\hline miRNA family & control nutrition (RPM) & Pi deficiency (RPM) & fold change \\
\hline miR398 & 21 & 94 & 2.0 \\
miR399 & 1.2 & 3.8 & 2.2 \\
miR408 & 1.4 & 6.8 & 2.3 \\
\hline
\end{tabular}

We identified one miRNA in the miR399 family (lus-miR399h UGCCAAAGGAGAGUUGCCCUG), one in the miR408 family (lus-miR408a AUGCACUGCCUCUUCCCUGGC) and three miRNAs in the miR398 family (lus-miR398d UGUGUUCUCAGGUCACCCCUC, lus-miR398b UGUGUUCUCAGGUCACCCCU, lus-miR398a UGUGUUCUCAGGUCGCCCCUG). Within the miR398 family only lus-miR398d was differentially expressed (3.8-fold change; p-value $2.4 * 10^{-7}$ ).

\subsection{Role of miR398 in Flax Pi Homeostasis Regulation}

miR398 is a conserved miRNA that participates in response to a combination of different stresses. In plants it was demonstrated that the miR398 family is involved in nutrient homeostasis regulation. miR398 participates in inorganic phosphate homeostasis (Zhu, Ding, \& Liu, 2011). In Arabidopsis miR398 is down-regulated in response to Pi starvation leading to increase in its target $\mathrm{CSD}(\mathrm{Cu} / \mathrm{Zn}$ superoxide dismutase) activity (Pant, Musialak-Lange, Nuc, May, Buhtz, Kehr, Walther, \& Scheible, 2009). At the same time, in tomato leaves it was shown that miR398 is up-regulated under Pi starvation conditions ( $\mathrm{Gu}, \mathrm{Xu}$, Chen, Zhu, Tang, \& Xu, 2010). miR398 is also involved in copper homeostasis by regulation of the expression of CCS (copper chaperone for superoxide dismutase), CSD1 (copper/zinc superoxide dismutase 1), CSD2 (copper/zinc superoxide dismutase 2) and FSD1 (Fe superoxide dismutase 1). In Arabidopsis miR398 is up-regulated during copper deficiency (Zhu et al., 2011) and down-regulated under $\mathrm{N}$ starvation conditions (Liang, He, \& Yu, 2012). Lus-miR398 targets were predicted in flax; their functions were: metabolism, signaling, transport and transcription factor (Neutelings, Fenart, Lucau-Danila, \& Hawkins, 2012; Barvkar et al., 2013). In our investigation of flax miRNAs, the up-regulation of miR398 under nutrient deficiency was observed. The same tendency in miR398 expression changes was shown for tomatoes $(\mathrm{Gu}$ et al., 2010). These alterations could be associated with Pi starvation and indicate a complex role of miR398 in plants nutrient homeostasis regulation. Flax miR398 could also influence the formation of the phenotype or genotype changes in response to Pi deficiency, but further studies of this miRNA expression in different plants under phosphate starvation are required for more precise conclusions.

\subsection{MiR408 Up-Regulation in Flax Under Pi Deficiency}

miR408 has been identified in more than 20 plant species, and it is one of the most conserved miRNA plant families, which indicates its importance. Different environmental stresses activate the signal pathways that involve induction of miR408 expression to modulate growth and development. miR408 is induced in response to copper deficiency, mechanical stress, dehydration, drought and reactive oxygen species (Zhang \& Li, 2013). It was shown that miR408 was also responsive to phosphate limitation and became more abundant during phosphate limitation (Pant et al., 2009). Functions of lus-miR408 predicted targets in flax were metabolism, transport and transcription factor (Neutelings et al., 2012). In our study we observed miR408 up-regulation induced by Pi deficiency, and this could indicate that this miRNA takes part in expression regulation during mineral nutrient stress in flax.

\subsection{MiR399 Role Pi Homeostasis Maintenance}

miR399 plays an important role in phosphate homeostasis. Pi starvation has resulted to up-regulation of miR399, and it negatively regulates the expression of ubiquitin-conjugating enzyme E2, which is the key element of the ubiquitination reaction (Jentsch, 1992). Also, it was shown that miR399 is a phloem-mobile long-distance signal for Pi homeostasis maintenance (Pant, Buhtz, Kehr, \& Scheible, 2008). The predicted target for lus-miR399 in flax 
is CDK (cyclin-dependent kinases) (Barvkar et al. 2013). In flax plants grown under nutrient-deprived conditions, miR399 expression was induced, suggesting a role for this miRNA family in Pi homeostasis regulation.

\section{Conclusions}

Using high-throughput sequencing for flax cultivar 'Stormont Cirrus', for the first time we identified miRNAs that were up-regulated in flax under Pi starvation. We demonstrated that expression level alterations of miR399 and miR408 in flax under Pi deficiency were close to those in other plant species. Flax miR398 was up-regulated under Pi starvation, the same was observed for tomato miR398, while in Arabidopsis miR398 was down-regulated in response to inorganic phosphate deficiency. These alterations could indicate a complex role of miR398 in plants phosphate homeostasis regulation. For better understanding of functions of miRNAs in plants under Pi deficiency conditions further investigations are needed. It could provide new tools for genetic improvement of stress tolerance in flax and other plants.

\section{Acknowledgments}

The work was financially supported by grants MK-6205.2012.4, RFBR 12-04-01469 and RFBR 13-04-01770.

\section{References}

Audic, S., \& Claverie, J. M. (1997). The significance of digital gene expression profiles. Genome Res, 7, $986-995$. http://dx.doi.org/10.1101/gr.7.10.986.

Barvkar, V. T., Pardeshi, V. C., Kale, S. M., Qiu, S., Rollins, M., Datla, R., ... Kadoo, N. Y. (2013). Genome-wide identification and characterization of microRNA genes and their targets in flax (Linum usitatissimum): Characterization of flax miRNA genes. Planta, 237, 1149-1161. http://dx.doi.org/10.1007/s00425-012-1833-5.

Bickel, C. L., Lukacs, M., \& Cullis, C. A. (2012). The loci controlling plasticity in flax. Res and Reports in Biol, 3, 1-11. http://dx.doi.org/10.2147/RRB.S27198.

Chen, Y., Lowenfeld, R., \& Cullis, C. A. (2009). An environmentally induced adaptive (?) insertion event in flax. Int J Genet Mol Biol, 1(3), 38-47.

Chen, Y., Schneeberger, R. G., \& Cullis, C. A. (2005). A site-specific insertion sequence in flax genotrophs induced by environment. New Phytol, 167, 171-180. http://dx.doi.org/10.1111/j.1469-8137.2005.01398.x

Cullis, C. (2007). Flax. In C. Kole (Ed.), Genome Mapping and Molecular Breeding in Plants, Volume 2 Oilseeds (pp. 275-295). Heidelberg, Germany, Springer Berlin Heidelberg.

Cullis, C. A., \& Charlton, L. (1981). The induction of ribosomal DNA changes in flax. Plant Sci. Lett., 20, $213-217$. http://dx.doi.org/10.1016/0304-4211(81)90264-9.

Durrant, A. (1958). Environmental conditioning of flax. Nature, 181, 928-929, http://dx.doi.org/10.1038/181928a0

Durrant, A. (1962). The environmental induction of heritable change in Linum. Heredity, 17, 27-61. http://dx.doi.org/10.1038/hdy.1962.2.

Evans, G. M., Durrant, A., \& Rees, H. (1966). Associated nuclear changes in the induction of flax genotrophs. Nature, 212, 697-699. http://dx.doi.org/10.1038/212697a0.

Gu, M., Xu, K., Chen, A., Zhu, Y., Tang, G., \&Xu, G. (2010). Expression analysis suggests potential roles of microRNAs for phosphate and arbuscular mycorrhizal signaling in Solanum lycopersicum. Physiol Plant, 138, 226-237. http://dx.doi.org/10.1111/j.1399-3054.2009.01320.x.

Guleria, P., Mahajan, M., Bhardwaj, J., \& Yadav, S. K. (2011). Plant small RNAs: biogenesis, mode of action and their roles in abiotic stresses. Genomics, Proteomics \& Bioinformatics, 9, $183-199$. http://dx.doi.org/10.1016/S1672-0229(11)60022-3.

Hoagland, D. R., \& Arnon, D. I. (1950). The water-culture method for growing plants without soil. California Agricultural Experiment Station Circular, 347, 1-32,

Jentsch, S. (1992). "The ubiquitin-conjugation system. Annu Rev Genet, 26, 179-207, http://dx.doi.org/10.1146/annurev.ge.26.120192.001143.

Jones-Rhoades, M. W., Bartel, D. P., \& Bartel, B. (2006). MicroRNAS and their regulatory roles in plants." Annual Review of Plant Biology, 57, 19-53. http://dx.doi.org/10.1146/annurev.arplant.57.032905.105218 
Kozomara, A., \& Griffiths-Jones, S. (2011). miRBase: integrating microRNA annotation and deep-sequencing data. Nucleic Acids Research, 39(Database issue), D152-157. http://dx.doi.org/10.1093/nar/gkq1027.

Liang, G., He, H., \& Yu, D. (2012). Identification of nitrogen starvation-responsive microRNAs in Arabidopsis thaliana. PLoS One, 7, e48951. http://dx.doi.org/10.1371/journal.pone.0048951

Neutelings, G., Fenart, S., Lucau-Danila, A., \& Hawkins, S. (2012). Identification and characterization of miRNAs and their potential targets in flax. Journal of Plant Physiology, 169, 1754-1766. http://dx.doi.org/10.1016/j.jplph.2012.06.011.

Pant, B. D., Buhtz, A., Kehr, J., \& Scheible, W. R. (2008). MicroRNA399 is a long-distance signal for the regulation of plant phosphate homeostasis. The Plant Journal: for Cell and Molecular Biology, 53, 731-738, http://dx.doi.org/10.1111/j.1365-313X.2007.03363.x.

Pant, B. D., Musialak-Lange, M., Nuc, P., May, P., Buhtz, A., Kehr, J., Walther, D., \& Scheible, W. R. (2009). Identification of nutrient-responsive Arabidopsis and rapeseed microRNAs by comprehensive real-time polymerase chain reaction profiling and small RNA sequencing. Plant Physiol, 150, 1541-1555. http://dx.doi.org/10.1104/pp.109.139139.

Sunkar, R., Chinnusamy, V., Zhu, J., \& Zhu, J. K. (2007). Small RNAs as big players in plant abiotic stress responses and nutrient deprivation. Trends Plant Sci, 12, 301-309, http://dx.doi.org/10.1016/j.tplants.2007.05.001.

Tyson, H., \& Fieldes, M. A. (1982). Molecular-weight and net charge of peroxidase isozymes in F1 hybrids between L and S flax genotrophs. Biochem Genet, 20, 919-927.

Zhang, H., \& Li, L. (2013). SQUAMOSA promoter binding protein-like7 regulated microRNA408 is required for vegetative development in Arabidopsis. Plant J, 74, 98-109. http://dx.doi.org/10.1111/tpj.12107

Zhu, C., Ding, Y., \& Liu, H. (2011). MiR398 and plant stress responses. Physiol Plant, 143, 1-9. http://dx.doi.org/10.1111/j.1399-3054.2011.01477.x

\section{Copyrights}

Copyright for this article is retained by the author(s), with first publication rights granted to the journal.

This is an open-access article distributed under the terms and conditions of the Creative Commons Attribution license (http://creativecommons.org/licenses/by/3.0/). 